\title{
Dispersion of the magnetostatic volume waves in a medium with damping
}

\author{
Lidiya Maltceva $^{1, \star}$, Pavel Makarov ${ }^{1, \star \star}$, Leonid Kotov ${ }^{1}$, and Vladimir Shcheglov ${ }^{2, \star \star \star}$ \\ ${ }^{1}$ Pitirim Sorokin Syktyvkar State University, Oktyabrsky 55, Syktyvkar, 167001, Russia \\ ${ }^{2}$ Institute of Radio-Engineering and Electronics of RAS, Mokhovaya 11-7, Moscow, 125009, Russia
}

\begin{abstract}
In magnetostatic approximation of electrodynamics the dispersion relation for magnetostatic backward volume waves (MSBVW) in a ferrite film, placed in a constant longitudinal and transverse alternating magnetic field is obtained. The magnetic field, damping and temperature influence on the spectrum and dispersion characteristics of the MSBVW is regarded.
\end{abstract}

\section{Introduction}

It is well known that spin waves have an extremely rich and peculiar dispersion that is nonlinear, anisotropic, and nonreciprocal $[1,2]$. The spin wave dispersion is very sensitive to the sample's magnetic properties and micromagnetic state, including both the internal magnetic field and magnetization. Nowadays, creating devices on magnetostatic surface (MSSW) and volume (MSVW) waves is one of the most actively developing areas of spintronics [3]. The use of monocrystalline ferro- and ferrimagnetic structures, such as iron-yttrium garnet (YIG) films, in systems based on MSVW allows the creation of compact devices with unique magnetic properties [4-9].

One of the main requirements for spintronics devices is the thermal stabilization of their magnetic parameters in the operating temperature range. At the same time, it is well known that YIG films have a serious drawback — the strong temperature dependence of the saturation magnetization.

Minimization of the energy losses is the key to the successful operation of spintronics devices, but MSVW attenuation and temperature instability has not been studied enoung, because most of the research are carried out without taking into account the attenuation and the effect of temperature [10].

\section{Model and basic equations}

The geometry of the problem is shown in Figure 1. Let's consider a boundless ferritic film of thickness $d$, magnetized to saturation. The plane $y \mathrm{Oz}$ corresponds to the plane of the film, the positions of the boundaries are determined by the coordinates $x= \pm d / 2$. The film is magnetized to saturation by a constant field $\mathbf{H}$ applied along the $O z$ axis. The angle between the $O y$ axis and the wave vector $\mathbf{k}$ is denoted as $\varphi$.

\footnotetext{
$\star$ e-mail: lidija.malceva@ rambler.ru

$\star \star$ e-mail: makarovpa@syktsu.ru

$\star \star \star$ e-mail: vshcheg@cplire.ru
}

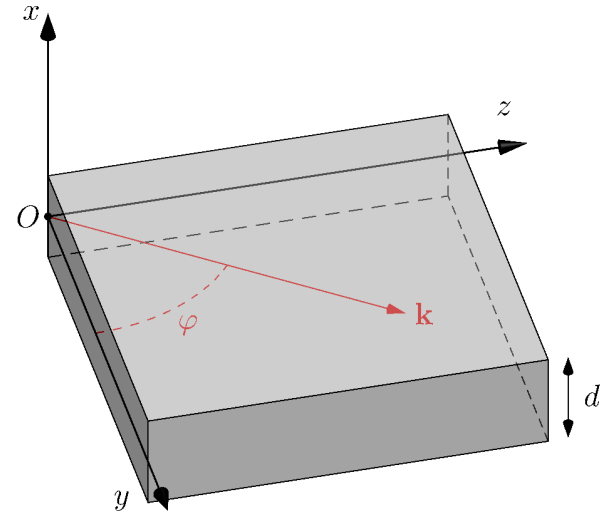

Figure 1. Geometry of the problem

Despite the fact that magnetostatics is only an approximation of electrodynamics, it can be used to model the work of a variety of spintronics devices. In the magnetostatic approximation Maxwell's magnetic equations have the form [11]

$$
\left\{\begin{array}{l}
\nabla \times \mathbf{H}=0, \\
\nabla \cdot(\mathbf{H}+4 \pi \mathbf{M})=0 .
\end{array}\right.
$$

If we introduce the magnetostatic potential $\psi$ :

$$
\mathbf{H}=\nabla \psi,
$$

then from (1) we obtain the Walker equation

$$
\Delta \psi+4 \pi \nabla \cdot \mathbf{M}=0 .
$$

The Landau-Lifshitz equation with a dissipative term in the Gilbert form was used to calculate the magnetization dynamics

$$
\frac{\partial \mathbf{M}}{\partial t}=-\gamma[\mathbf{M} \times \mathbf{H}]+\frac{\alpha}{M_{0}}\left[\mathbf{M} \times \frac{\partial \mathbf{M}}{\partial t}\right]
$$


In (4) $M_{0}$ is the constant length of the magnetization vector, $\gamma$ is the gyromagnetic ratio, and $\alpha$ is the dissipation parameter. The dispersion equation for the magnetostatic volume waves, obtained from (3) and (4), has the form:

$$
\tan (k d \vartheta)=\frac{2 \mu \vartheta}{\beta-1}
$$

where $\vartheta, \beta-$ ancillarity quantities:

$$
\vartheta=\sqrt{\cos ^{2} \varphi+\frac{\sin ^{2} \varphi}{\mu}}, \quad \beta=v^{2} \cos \varphi-\mu^{2} \vartheta^{2} .
$$

In (6) $\mu$ is the permeability and $v$ is the magnetic susceptibility of the medium

$$
\begin{aligned}
& \mu=1+\frac{\Omega_{H}+i \alpha \Omega}{\Omega_{H}^{2}-\left(1+\alpha^{2}\right) \Omega^{2}+i 2 \alpha \Omega \Omega_{H}}, \\
& \nu=\frac{\Omega}{\Omega_{H}^{2}-\left(1+\alpha^{2}\right) \Omega^{2}+i 2 \alpha \Omega \Omega_{H}},
\end{aligned}
$$

which depend of the normalized magnetic field $\Omega_{H}$ and normalized frequency $\Omega$ :

$$
\Omega_{H}=\frac{H}{4 \pi M_{0}}, \quad \Omega=\frac{\omega}{4 \pi \gamma M_{0}} .
$$

\section{Main results}

The dispersion curves for the first five modes of the magnetostatic volume waves in the case when the angle $\varphi=\pi / 2$ without damping $(\alpha=0)$ are shown in Figure 2.

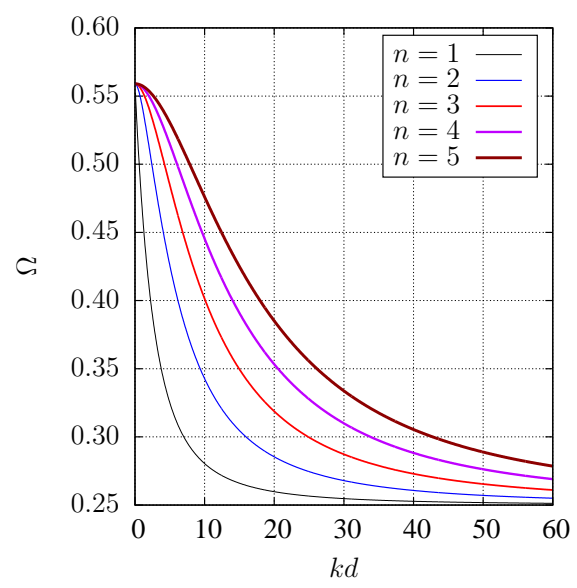

Figure 2. Dispersion curves for first five modes of MSBVW without damping in case $\varphi=\pi / 2$.

The analysis of dispersion curves (Figure 2) shows, that in our geometry (Figure 1) only the magnetostatic backward volume waves (MSBVW) can exist.

The boundary frequencies of the MSBVW, defined from the limit transition $k \longrightarrow 0$ and $k \longrightarrow \infty$ of the dispersion relation (5) have the form:

$$
\left\{\begin{array}{l}
f_{1}=\gamma H \\
f_{2}=\gamma \sqrt{H\left(H+4 \pi M_{0}\right)} .
\end{array}\right.
$$

Thus, the volume waves propagate in a strictly defined frequency range $f_{1} \leqslant f \leqslant f_{2}$. The boundary frequencies of the MSBVW are determined by the value of the saturation magnetization and can vary over a wide range. At the same time, the saturation magnetization of the material substantially depends on the temperature.

\subsection{Influence of the constant magnetic field value and the direction of propagation on the dispersion of MSBVW}

The curves of the boundary frequencies as functions of a constant magnetic field are shown in Figure 3. In addition, this figure shows the field dependences of the width of the MSBVW frequency range (dashed line).

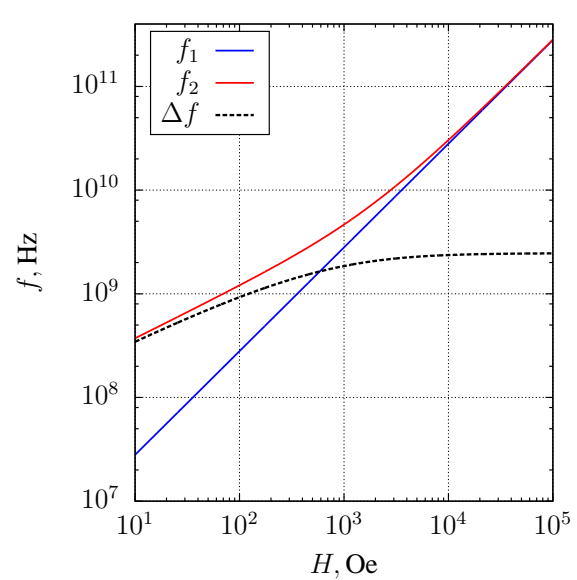

Figure 3. Magnetic field effect on the MSBVW spectrum

Figure 3 illustrates that in weak fields the lower boundary frequency of the MSBVW, in contrast to the upper one, linearly depends on the field. However, in the limiting transition $H \rightarrow \infty$ both of $f_{1}$ and $f_{2}$ are linearly dependent on $H$. Thus, with an increase in the magnetic field from 10 Oe to about $10 \mathrm{kOe}$, the frequency spectrum of MSBVW expands from about $0.5 \mathrm{GHz}$ to $2 \mathrm{GHz}$. In fields $H$ more than $10 \mathrm{kOe}$, the width of the spectrum does not change and depends on the saturation magnetization.

The influence of the angle $\varphi$ on the boundary frequencies is shown in Figure 4. From this figure we can see that the high frequency of MSBVW spectrum $f_{2}$ equals to the FMR frequency and does not depend on angle $\varphi$. The low boundary frequency of MSBVW spectrum $f_{1}$ is a decreasing function of angle between magnetic field and direction of wave propagation. Therefore, the MSBVW spectrum decreases with the reduction of angle $\varphi$.

Figure 5 shows the frequency dependence of the first mode of the MSBVW for the real part of the wave number at different angles in case when damping is negligible. The angle change leads to the alteration of the spectrum width of the MSBVW.

It is evident that the frequency spectrum of the MSBVW expands with the increasing angle $\varphi$. The region of existence of volume waves is limited by the angle $\varphi \neq 0$. 


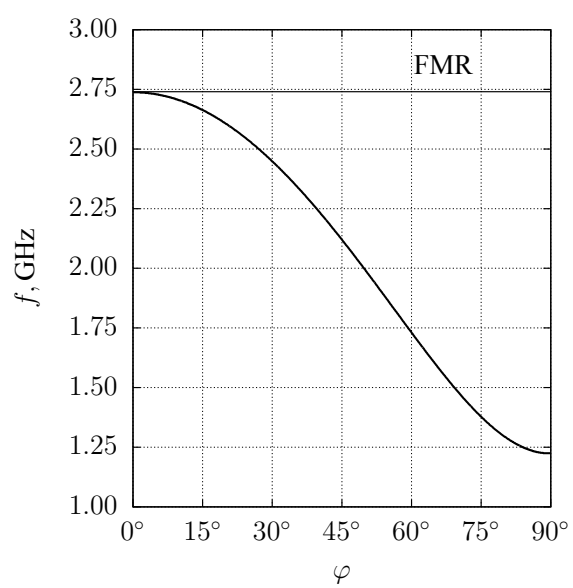

Figure 4. Angle effect on the MSBVW spectrum

\subsection{Temperature influence}

It is well-known that YIG films exhibit strong temperature instability, which can be caused by a number of factors [12]. In our model, the effect of temperature reduces to the fact that the normalized frequencies of the waves $\Omega$ and the magnetic fields $\Omega_{H}$, determined by the relations (8), shift up with changing $T$.

In the temperature range $T$ from $213 \mathrm{~K}$ to $353 \mathrm{~K}$ the function $4 \pi M(T)$ can be approximated by a linear dependence, and tangent of the slope angle is equal to $-2.9 \mathrm{G} / \mathrm{K}$ [12]. Thus, with the temperature decreasing from $T=300 \mathrm{~K}$ to $T=273 \mathrm{~K}$, the saturation magnetization increases from $1760 \mathrm{G}$ to $1840 \mathrm{G}$.

Dispersion curves for the first five modes of the MSBVW at different temperatures $(T=273 \mathrm{~K}$ and $T=300 \mathrm{~K}$ ) are shown in Figure 6.

It is clear from Figure 6(a) and Figure 6(b) that changing of the temperature alterates the dispersion curves slightly, in particular, with increasing $T$ the frequency spectrum shifts to a lower frequency region.

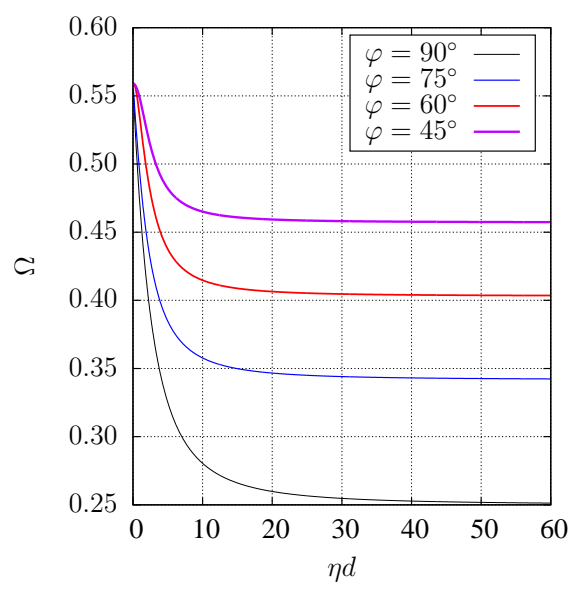

Figure 5. The angle influence on the dispersion of first mode of MSBVW

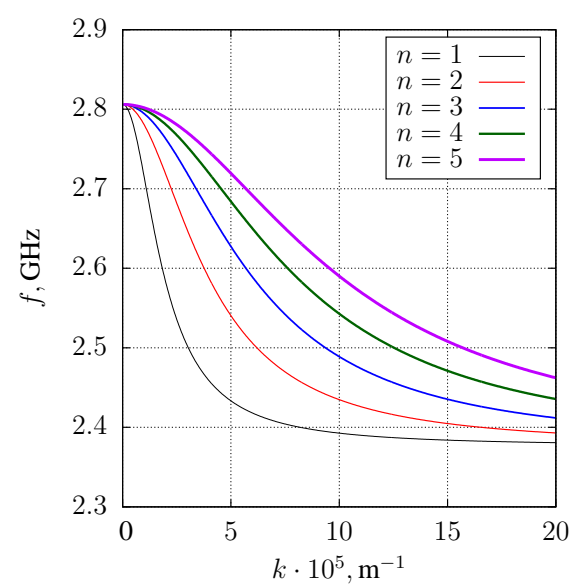

(a) $T=273 \mathrm{~K}$

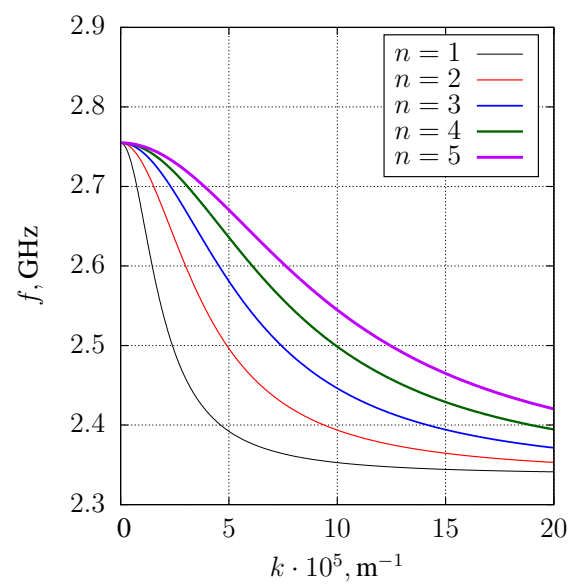

(b) $T=300 \mathrm{~K}$

Figure 6. Dispersion curves for the first five modes of MSBVW

In Figures 6(a), and 6(b) the follows parameters were used: film thickness $d=15 \mathrm{mkm}$, gyromagnetic ratio $\gamma=2$, magnetic field value $H=440$ Oe, angle $\varphi=40^{\circ}$. The magnetic field $H$ and angle $\varphi$ values were chosen as intermediate numbers between minimum and maximum quantities. This choice approximately corresponds to the average point of the dashed curve in Figure 3 and middle value of the spectrum width in Figure 4.

\subsection{The influence of dissipation}

Let's consider the case when $\alpha \neq 0$. In this case parameters $v, \mu, \beta, \vartheta$, as well as the wave vector $k=\eta-i \xi$ are complex values. The imaginary part $\xi$ of the wave vector determines the logarithmic damping decrement.

The left-hand side of the equation (5) is a function of both the wave vector $k$ and the frequency $\Omega$. However, with help of the auxiliary notation (10), it is easy to separate the variables.

$$
\mathcal{Z}=\arctan \frac{2 \mu \vartheta}{\beta-1}
$$


In this way, the dispersion relation (5) can be written as follows:

$$
\eta d=\frac{\mathcal{Z}^{\prime} \vartheta^{\prime}+\mathcal{Z}^{\prime \prime} \vartheta^{\prime \prime}}{\left(\vartheta^{\prime}\right)^{2}+\left(\vartheta^{\prime \prime}\right)^{2}}, \quad \xi d=\frac{\mathcal{Z}^{\prime} \vartheta^{\prime \prime}-\mathcal{Z}^{\prime \prime} \vartheta^{\prime}}{\left(\vartheta^{\prime}\right)^{2}+\left(\vartheta^{\prime \prime}\right)^{2}}
$$

In case of arbitrary geometry (for various angles $\varphi$ ) and when damping taking into account the dispersion of the MSBVW is complex.

Figure 7 illustrates the effect of damping on the dispersion characteristics of the first five modes of the MSBVW. This effect leads to the fact that the wave number is limited both from above (in the low-frequency region) and from below (in the high-frequency region). Also, it is clear that damping, in addition to limiting the possible values of the wave number (and hence also the wavelength of the MSBVW), affects the shape of the dispersion curves. This means that the presence of attenuation changes the propagation velocity of the waves.

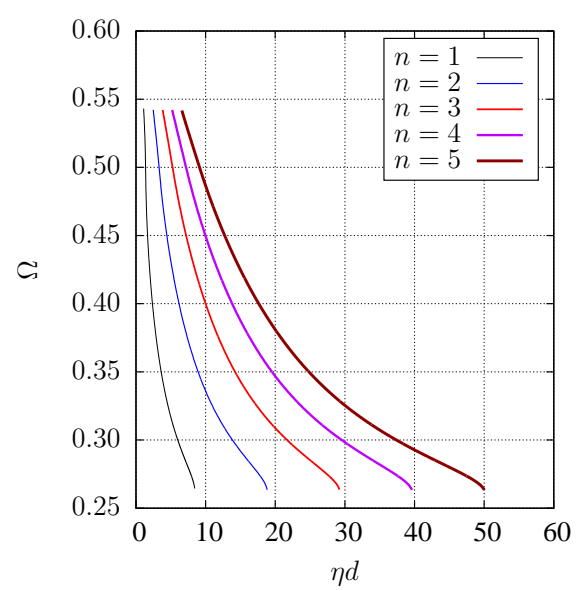

Figure 7. Dispersion curves of the first five modes of the MSBVW when $\alpha=0.05$ for $\varphi=\pi / 2$

\section{Conclusion}

Thus, the analysis of dispersion curves with and without of damping, showed that dissipation leads to the limi- tation of the spectrum of MSBVW not only in frequency, but also in wavelength.

Moreover, field and angular dependences of the boundary frequencies and spectrum width for magnetostatic backward volume waves are obtained. In addition, the effect of temperature on the dispersion curves of the MSBVW is determined: with the increase of the temperature, the frequency spectrum shifts to a lower frequency region.

This research was supported by Russian Scientific Fund (projects 17-02-01138 and 17-57-150001).

\section{References}

[1] S.O. Demokritov Spin Wave Confinement: Propagating Waves (2nd Edition) (Pan Stanford Publishing Pte. Ltd., Singapore, 2017) 449

[2] D. Stancil, A. Prabhakar Spin Waves. Theory and Applications (Springer, New York, 2009) 364

[3] A.V. Sadovnikov et al. Phys. Rev. B 96, 060401(R) (2017)

[4] J.P. Parekh, K.W. Chang, H.S. Tuan, Circuits, Systems and Signal Processing 4, 9-39 (1985)

[5] W.S. Ishak, Proceedings of the IEEE 76, 171-187 (1988)

[6] K.L. Wang, Z. Zhao, A. Khitun, Thin Solid Films 517, 184-190 (2008)

[7] A.A. Serga, A.V. Chumak, B. Hillebrands, J. Phys. D: Appl. Phys. 43, 264002 (2010)

[8] A. Khitun, M.Q. Bao, K.L. Wang, J. Phys. D: Appl. Phys., 43, 264005 (2010)

[9] A.V. Chumak, V.I. Vasyuchka, A.A. Serga, B. Hillebrands, Nat. Phys., 11, 453 (2015)

[10] R.W. Damon, J.R. Eshbach, J. Phys. Chem. Solids 19, 308 (1961)

[11] L.D. Landau, E.M. Lifshitz The Classical Theory of Fields (Pergamon Press, Oxford, 1971) 430

[12] S.I. Yushchuk, P.S. Kostyuk, I.E. Lopatinskii, Tech. Phys. The Russian J. of Appl. Phys. 43, 1051-1054 (1998) 